\title{
Coronary artery bypass in patients with type 2 diabetes: Experience from the Bypass Angioplasty Revascularization Investigation 2 Diabetes trial
}

\author{
Edward Y. Sako, MD, PhD, ${ }^{a}$ Maria Mori Brooks, PhD, ${ }^{\mathrm{b}}$ Regina M. Hardison, MS, \\ Hartzel Schaff, $\mathrm{MD},{ }^{\mathrm{c}}$ and Robert L. Frye, $\mathrm{MD}^{\mathrm{c}}$
}

Objective: Bypass Angioplasty Revascularization Investigation 2 Diabetes (BARI 2D) was a study of management
strategies for diabetic patients with myocardial ischemia and coronary artery disease. In a $2 \times 2$ design, early
revascularization versus medical management with or without late revascularization and insulin sensitization versus
insulin provision were examined. No advantage for either strategy was seen, except in the group undergoing early
coronary artery bypass grafting (CABG). In that group, a reduction in subsequent myocardial infarction was noted.
The purpose of our report was to characterize the conduct and short-term outcomes for CABG that led to this result.

\begin{abstract}
Methods: Data from the BARI 2D CABG stratum were collected, including the baseline demographic and cardiovascular characteristics, technical details of the operation, and perioperative morbidity and mortality, and analyzed.
\end{abstract}

\begin{abstract}
Results: A total of 347 patients were studied. The average cardiac function was normal, and most had multivessel disease. Almost all had undergone CABG by way of a median sternotomy using an internal mammary artery, and one third were off pump. The perioperative morbidity and mortality were low and compared well with larger outcomes databases.
\end{abstract}

Conclusions: BARI 2D showed that early CABG in patients with type 2 diabetes and myocardial ischemia and multivessel disease reduced the subsequent myocardial infarction rates. The present results have demonstrated that this was achieved using off-pump surgery in certain cases, standard myocardial protection, and routine use of the internal mammary artery or other arterial grafts. (J Thorac Cardiovasc Surg 2014;148:1268-72)

The association between coronary artery disease (CAD) and type 2 diabetes mellitus (DM) is well known. The original Bypass Angioplasty Revascularization Investigation (BARI) trial, which had included patients who had

\footnotetext{
From the University of Texas Health Science Center at San Antonio, ${ }^{a}$ San Antonio, Tex; University of Pittsburgh, ${ }^{\mathrm{b}}$ Pittsburgh, Pa; and Mayo Clinic, ${ }^{\mathrm{c}}$ Rochester, Minn. The Bypass Angioplasty Revascularization Investigation 2 Diabetes (BARI 2D) was funded by the National Heart, Lung, and Blood Institute and the National Institute of Diabetes and Digestive and Kidney Diseases (grants U01 HL061744, U01 HL061746, U01 HL061748, and U01 HL063804) with significant supplemental funding from GlaxoSmithKline (Collegeville, Pa), Lantheus Medical Imaging, Inc (formerly Bristol-Myers Squibb Medical Imaging, North Billerica, Mass), Astellas Pharma US, Inc (Deerfield, Ill), Merck \& Co, Inc (Whitehouse Station, NJ), Abbott Laboratories, Inc (Abbott Park, Ill), and Pfizer, Inc (New York, NY). Generous support has been given by Abbott Laboratories Ltd, MediSense Products (Mississauga, Ontario, Canada), Bayer Diagnostics (Tarrytown, NY), Becton Dickinson and Co (Franklin Lakes, NJ), J.R. Carlson Laboratories (Arlington Heights, Ill), Centocor, Inc (Malvern, Pa), Eli Lilly and Co (Indianapolis, Ind), LipoScience, Inc (Raleigh, NC), Merck Sante (Lyon, France), Novartis Pharmaceuticals Corp (East Hanover, NJ), and Novo Nordisk, Inc (Princeton, NJ).

Disclosures: Edward Y. Sako reports lecture fees from Edwards and equity ownership in Medtronic. All other authors have nothing to disclose with regard to commercial support.

Received for publication Oct 1, 2013; revisions received Nov 16, 2013; accepted for publication Nov 30, 2013; available ahead of print Feb 4, 2014.

Address for reprints: Edward Y. Sako, MD, PhD, Department of Cardiothoracic Surgery, University of Texas Health Science Center at San Antonio, Mail Code 7841, 7703 Floyd Curl Dr, San Antonio, TX 78229-3900 (E-mail: sako@uthscsa.edu). $0022-5223 / \$ 36.00$

Copyright (c) 2014 by The American Association for Thoracic Surgery http://dx.doi.org/10.1016/j.jtcvs.2013.11.038
}

undergone revascularization late in their course of $\mathrm{CAD}$, demonstrated a dramatic reduction in survival among those with DM compared with those without DM, regardless of whether they had undergone percutaneous transluminal coronary angioplasty or coronary artery bypass grafting (CABG). ${ }^{1}$ Among the patients with $\mathrm{DM}$, a significant benefit was found for CABG, in particular, with an internal mammary graft, relative to percutaneous transluminal coronary angioplasty. However, it was noted that patients receiving insulin had a worse outcome than those requiring oral agents only to control their DM. BARI 2 Diabetes (BARI 2D) was thus planned to determine the treatment strategies that might reduce the excess mortality associated with CAD in patients with type 2 DM. Early revascularization versus optimal medical management with delayed or no revascularization and insulin sensitization versus insulin provision to achieve glycemic control were tested in a $2 \times 2$ design. No advantage was documented for either strategy in the primary analysis. However, within the CABG stratum, a significant benefit for $\mathrm{CABG}$ in reducing subsequent myocardial infarction (MI) was observed. ${ }^{2}$ In the present report, we have described the methods used in the conduct of CABG in the BARI 2D centers to better place this important finding into context. 


\section{Abbreviations and Acronyms \\ BARI = Bypass Angioplasty Revascularization Investigation \\ BARI 2D = Bypass Angioplasty Revascularization Investigation 2 Diabetes \\ $\mathrm{CABG}=$ coronary artery bypass grafting \\ $\mathrm{CAD}=$ coronary artery disease \\ $\mathrm{DM} \quad=$ diabetes mellitus \\ IMA $=$ internal mammary artery \\ MI = myocardial infarction \\ PCI = percutaneous coronary intervention}

\section{METHODS}

The details of the design of BARI 2D have been previously presented. ${ }^{1}$ The trial was sponsored by the National Institutes of Health, with additional support from industry. The industry sponsors did not have access to the outcomes data and did not participate in the data analysis or preparation of our report. The institutional review board at each participating site approved the protocol (ClinicalTrials.gov NCT00006305). All patients provided written informed consent. An independent data and safety monitoring board approved the study protocol and monitored the conduct of the study and safety of the patients.

A $2 \times 2$ factorial design was used to randomly assign patients to 2 treatment strategies. In the first strategy, the patients were randomized to early (within 4 weeks) revascularization or medical therapy. In the second strategy, the patients were assigned to either insulin-sensitization therapy or insulin-provision therapy. The target for these therapies was a glycated hemoglobin level of $<7.0 \%$.

Before randomization, the patients were assigned to a stratum of percutaneous coronary intervention (PCI) or CABG as the method of coronary revascularization by the physician responsible using the clinical criteria in place at each respective institution. This was not meant to be a study of CABG versus PCI but, rather, a real world application of the revascularization strategy. Patients in the medical therapy arm underwent subsequent revascularization during follow-up if they had progression of angina or had developed acute coronary syndrome or acute myocardial ischemia. Each participating institution was required to adhere to specific surgical requirements as set forth by the BARI 2D trial.

For the purpose of the present analysis and to reflect the results of the BARI 2D trial, we focused on the patients randomized to early revascularization, who had undergone $\mathrm{CABG}$ as their initial revascularization procedure.

The conduct of the operation and perioperative care was left to the individual surgeons and their institutional protocols. The decision to perform a case on pump versus off pump was determined by the perceived quality of the targets and surgeon preference. Similarly, the myocardial protection schemes in the on-pump group were left to the surgeon's discretion. The use of $\geq 1$ internal mammary arteries was not mandated but was encouraged. Incomplete revascularization was allowed, but it was expected that all stenosis contributing to the patient's clinical symptoms and areas of ischemia would be bypassed. The management of insulin use in the perioperative period was not mandated by a specific central protocol but was left to the individual centers to use their established protocols, noting the importance of attention to glycemic control in the perioperative period.

In addition to recording the number of diseased vessels, the myocardial jeopardy index was calculated for each patient. This is the ratio of jeopardized and anatomically relevant segments downstream of significant lesions relative to all viable left ventricular segments and is reported as a percentage. ${ }^{3}$ A multivariate analysis of the surgery was performed that included the baseline characteristics, cardiovascular and DM factors on presentation, conduct of the operation, and outcomes.

\section{RESULTS}

Of the 1176 patients assigned to prompt revascularization in the BARI 2D trial, 95.4\% had undergone revascularization within 6 months of entry. A total of 347 patients underwent $\mathrm{CABG}$ as their initial assigned revascularization. The present report describes the results for that group of patients.

The demographic characteristics of the 347 patients who were assigned to revascularization and underwent CABG are listed in Table 1. Of these patients, $98 \%$ were from the CABG stratum (ie, CABG was the intended method revascularization before randomization). Their mean age was 63 years, and $75 \%$ were men. The BARI $2 \mathrm{D}$ trial was an international multicenter study; $40 \%$ of the CABG patients were from the United States, $32 \%$ from Brazil, 17\% Canada, 7\% Mexico, and 5\% from Central Europe. Most of the patients were white; however, black and Hispanic patients were well-represented in the CABG cohort.

The cardiovascular clinical history is presented in Table 2. One third of the patients had had a previous MI and $8 \%$ a previous cerebrovascular accident or TIA. Within 6 weeks of study entry, $58 \%$ were classified as having stable angina and $10 \%$ unstable angina. Of the patients, $12 \%$ had undergone previous PCI, and just more than one half of these had received a stent. Only 1 patient had undergone previous CABG. The average ejection fraction was $58 \%$, with less than one fifth of patients having an abnormal ejection fraction, as defined by a left ventricular ejection fraction $<50 \%$. Most patients in the present cohort had multivessel disease, defined as $\geq 2$ major vessels with $\geq 50 \%$ stenosis.

TABLE 1. Baseline characteristics $(n=347)$

\begin{tabular}{lc}
\hline \multicolumn{1}{c}{ Characteristic } & Value \\
\hline CABG stratum (\%) & 97.7 \\
Male gender (\%) & 74.9 \\
Mean age at study entry (y) & $62.9 \pm 8.6$ \\
Age category (\%) & \\
<50 y & 8.1 \\
50-59 y & 27.7 \\
60-69 y & 43.2 \\
$\geq 70$ y & 21.0 \\
Race/ethnicity (\%) & \\
White, non-Hispanic & 69.7 \\
Black, non-Hispanic & 11.8 \\
Hispanic & 14.4 \\
Asian, non-Hispanic & 4.0 \\
Geographic region (\%) & \\
United States & 39.5 \\
Canada & 16.7 \\
Mexico & 7.2 \\
Brazil & 31.7 \\
Czech Republic/Austria & 4.9 \\
\hline
\end{tabular}

$C A B G$, Coronary artery bypass grafting. 
TABLE 2. Baseline cardiovascular characteristics $(n=347)$

\begin{tabular}{|c|c|}
\hline Characteristic & Value \\
\hline History of MI & 34.5 \\
\hline History of CHF requiring therapy & 4.6 \\
\hline CVA or TIA & 7.8 \\
\hline Extracoronary artery disease & 24.8 \\
\hline Carotid disease & 9.8 \\
\hline History of peripheral vascular surgery & 2.0 \\
\hline Intermittent claudication & 18.4 \\
\hline Abdominal aneurysm & 0.9 \\
\hline COPD & 3.5 \\
\hline Pulmonary edema & 1.2 \\
\hline Asthma & 2.0 \\
\hline Chronic renal dysfunction & 2.6 \\
\hline History of malignancy (without nonmelanoma) & 6.9 \\
\hline \multicolumn{2}{|l|}{ Angina category within $6 \mathrm{wk}$} \\
\hline Stable CCS1 & 11.2 \\
\hline Stable CCS2 & 31.7 \\
\hline Stable CCS3 & 13.0 \\
\hline Stable CCS4 & 1.7 \\
\hline Unstable angina & 10.1 \\
\hline Anginal equivalents but no angina & 17.0 \\
\hline No angina nor anginal equivalents & 15.3 \\
\hline Site numeric LVEF information available & 97.7 \\
\hline Mean site LVEF (\%) & $57.6 \pm 11.5$ \\
\hline Site abnormal LVEF information available & 98.6 \\
\hline Site LVEF < $50 \%$ & 17.3 \\
\hline \multicolumn{2}{|l|}{ Angiographic core laboratory evaluation } \\
\hline Myocardial jeopardy score & $61.4 \pm 21.1$ \\
\hline Number of lesions $\geq 20 \%$ & $5.8 \pm 2.3$ \\
\hline Lesions $\geq 50 \%$ stenosis & $3.7 \pm 1.7$ \\
\hline Lesions $\geq 70 \%$ stenosis & $1.7 \pm 1.2$ \\
\hline \multicolumn{2}{|l|}{ Number of vessels with lesions $\geq 50 \%$} \\
\hline 1 & 13.9 \\
\hline 2 & 42.4 \\
\hline 3 & 43.8 \\
\hline Totally occluded lesions & 62.5 \\
\hline Proximal LAD $\geq 50 \%$ stenosis & 19.3 \\
\hline Previous $\mathrm{CABG}$ & 0.3 \\
\hline Lesions in graft vessels & 0.3 \\
\hline Previous PCI & 11.8 \\
\hline Previous stent & 6.3 \\
\hline In-stent lesion & 2.6 \\
\hline
\end{tabular}

Data presented as $\%$ or mean \pm standard deviation. MI, Myocardial infarction; $C V A$, cerebrovascular accident; TIA, transient ischemic attack; COPD, chronic obstructive pulmonary disease; $C C S$, Canadian Cardiovascular Society; $L V E F$, left ventricular ejection fraction; $L A D$, left anterior descending (artery); $C A B G$, coronary artery bypass grafting; $P C I$, percutaneous coronary intervention; $C H F$, congestive heart failure.

The baseline DM and metabolic characteristics are listed in Table 3. The mean duration of DM diagnosis was 10 years, and $22 \%$ of patients were taking insulin. This cohort had a mean glycated hemoglobin level of $7.7 \%$ and a mean body mass index of $30 \mathrm{~kg} / \mathrm{m}^{2}$.

The characteristics of the CABG procedures are listed in Table 4. Almost all (97\%) of the CABG procedures were performed electively and only $2(0.6 \%)$ as an emergency. On
TABLE 3. Baseline metabolic characteristics $(n=347)$

\begin{tabular}{|c|c|}
\hline Characteristic & Value \\
\hline Age at DM diagnosis (y) & $52.3 \pm 10.4$ \\
\hline Duration of DM (y) & $10.1 \pm 8.1$ \\
\hline Insulin use at baseline & 21.9 \\
\hline BMI $\left(\mathrm{kg} / \mathrm{m}^{2}\right)$ & $30.0 \pm 4.7$ \\
\hline \multicolumn{2}{|l|}{ BMI category } \\
\hline Low, $<20 \mathrm{~kg} / \mathrm{m}^{2}$ & 0.6 \\
\hline Normal, 20 to $<25 \mathrm{~kg} / \mathrm{m}^{2}$ & 13.0 \\
\hline Overweight, 25 to $<30 \mathrm{~kg} / \mathrm{m}^{2}$ & 40.3 \\
\hline Class 1 obesity, 30 to $<35 \mathrm{~kg} / \mathrm{m}^{2}$ & 30.7 \\
\hline Class 2 obesity, 35 to $<40 \mathrm{~kg} / \mathrm{m}^{2}$ & 12.8 \\
\hline Class $3-4$ obesity, $\geq 40 \mathrm{~kg} / \mathrm{m}^{2}$ & 2.6 \\
\hline Average systolic blood pressure ( $\mathrm{mm} \mathrm{Hg}$ ) & $131.7 \pm 21.0$ \\
\hline Average diastolic blood pressure (mm Hg) & $75.4 \pm 11.3$ \\
\hline Sitting blood pressure $>130 / 80 \mathrm{~mm} \mathrm{Hg}$ & 52.6 \\
\hline Sitting blood pressure $>140 / 90 \mathrm{~mm} \mathrm{Hg}$ & 29.5 \\
\hline Orthostatic hypotension & 14.0 \\
\hline Core $\mathrm{HbA} 1 \mathrm{c}$ measure available & 95.7 \\
\hline Core HbA1c $(\%)$ & $7.70 \pm 1.68$ \\
\hline \multicolumn{2}{|l|}{ Core HbA1c category } \\
\hline$<7$ & 38.3 \\
\hline $7-8$ & 22.6 \\
\hline$\geq 8$ & 39.2 \\
\hline Core lipid measures available & 98.0 \\
\hline Core total cholesterol $(\mathrm{mg} / \mathrm{dL})$ & $170.8 \pm 40.0$ \\
\hline Core triglycerides $(\mathrm{mg} / \mathrm{dL})$ & $179.1 \pm 99.5$ \\
\hline Core LDL (mg/dL) & $97.9 \pm 34.3$ \\
\hline Core HDL (mg/dL) & $37.5 \pm 9.5$ \\
\hline
\end{tabular}

average, the interval from randomization to surgery was 27 days. Just more than one third of the cases were done off pump. Also, regardless of whether the case was performed on or off pump, $99 \%$ underwent full sternotomy. Of the 221 cases done on pump, the mean duration of bypass was 91 minutes, and the mean crossclamp time was 60 minutes. As a method to achieve myocardial protection, blood cardioplegia was used in $69 \%$, crystalloid cardioplegia in $14 \%$, and intermittent crossclamp in 13\%. Retrograde cardioplegia was used in one quarter of the patients. An internal mammary artery (IMA) or IMA with a saphenous vein graft were used in $88 \%$ of the CABG procedures. In $15 \%$ of cases, another artery was used, and a saphenous vein graft alone was used in only $5 \%$ of the cases. The total number of grafts averaged 2.8 , with a mean number of distal anastomoses of 3.0.

The operative results are listed in Table 5. One intraoperative death occurred. Postoperatively, $3.5 \%$ of patients required an intra-aortic balloon pump, and $1.5 \%$ required a ventricular assist device. Within the index hospitalization, the occurrence of major cardiac, neurologic, and postoperative events was all very low. Of particular note, the incidence of perioperative MI, stroke, renal failure, and mediastinitis was $<1.5 \%$ for each. 
TABLE 4. CABG characteristics $(n=347)$

\begin{tabular}{|c|c|}
\hline Characteristic & Value \\
\hline Interval from randomization (d) & $27.15 \pm 25.56$ \\
\hline \multicolumn{2}{|l|}{ Revascularization priority } \\
\hline Elective & 97.4 \\
\hline Urgent & 2.0 \\
\hline Emergency & 0.6 \\
\hline \multicolumn{2}{|l|}{ Angina status before $\mathrm{CABG}$} \\
\hline Stable & 90.2 \\
\hline Unstable & 8.9 \\
\hline Acute MI & 0.9 \\
\hline Full sternotomy & 98.6 \\
\hline Partial sternotomy & 1.2 \\
\hline Lateral thoracotomy & 0.3 \\
\hline Procedure performed off pump & 36.0 \\
\hline \multicolumn{2}{|c|}{ Primary method of myocardial protection $(\mathrm{n}=221)$} \\
\hline Intermittent crossclamp & 13.1 \\
\hline Blood cardioplegia & 69.2 \\
\hline Crystalloid cardioplegia & 12.7 \\
\hline Oxygen crystalloid cardioplegia & 1.4 \\
\hline Continuous perfusion, no clamp & 3.6 \\
\hline Retrograde perfusion & 25.1 \\
\hline Topical hypothermia & 35.3 \\
\hline Lowest core temperature $\left({ }^{\circ} \mathrm{C}\right)$ & $32.72 \pm 3.19$ \\
\hline Bypass duration (min) & $90.64 \pm 44.78$ \\
\hline Crossclamp duration (min) & $60.42 \pm 29.02$ \\
\hline \multicolumn{2}{|l|}{ Artery used } \\
\hline IMA only & 7.8 \\
\hline SVG only & 5.2 \\
\hline IMA and SVG & 80.7 \\
\hline Other artery & 15.0 \\
\hline Other vein & 0.6 \\
\hline
\end{tabular}

The 30-day mortality for this group was $1.4 \%(\mathrm{n}=5)$. The occurrence of MI and stroke within 30 days was $2.9 \%(\mathrm{n}=10)$ and $0.3 \%(\mathrm{n}=1)$, respectively, and the composite of death, MI, and stroke was $4.6 \%$ at 30 days (Figure 1).

\section{DISCUSSION}

BARI 2D was designed to assess the management strategies after clinically indicated coronary angiography in patients with type $2 \mathrm{DM}$ and mild or no angina but evidence of ischemia on stress testing. With regard to the mode of revascularization, the BARI $2 \mathrm{D}$ trial was not designed as a randomized comparison of CABG versus PCI but to determine whether early revascularization compared with delayed or no revascularization reduced mortality and other events and whether the use of insulin-sensitizing agents rather than insulin would reduce the high event rates of those with DM. The design was to randomize the patients after the cardiologist had decided which mode of revascularization was in the best interest of the patient, and the
TABLE 5. Operative results $(n=347)$

\begin{tabular}{|c|c|}
\hline Characteristic & $\%$ \\
\hline Event in OR & 7.2 \\
\hline Cardiac arrest & 1.2 \\
\hline Suspected MI & 0.3 \\
\hline $\mathrm{CHF}$ & 0.3 \\
\hline Cardiogenic shock & 0.9 \\
\hline Hemorrhage requiring transfusion & 2.0 \\
\hline Hypersensitivity reaction & 0.6 \\
\hline Hypotension requiring treatment & 1.4 \\
\hline Reoperation for bleeding & 0.9 \\
\hline Event requiring transfusion & 0.3 \\
\hline Other event & 2.3 \\
\hline Death in OR & 0.3 \\
\hline \multicolumn{2}{|l|}{ Postoperative events } \\
\hline Inotropic agents during 48 -h period & 9.2 \\
\hline New permanent pacemaker & 0.6 \\
\hline Left ventricular assist device & 0.9 \\
\hline Right ventricular assist device & 0.6 \\
\hline Intra-aortic balloon pump use postoperatively & 3.5 \\
\hline Cardiac arrest & 0.3 \\
\hline Suspect MI & 1.2 \\
\hline $\mathrm{CHF}$ & 0.3 \\
\hline Pulmonary edema & 0.3 \\
\hline Cardiogenic shock & 0.3 \\
\hline Cardiac tamponade & 0.3 \\
\hline Hemorrhage requiring transfusion & 1.7 \\
\hline Hypotension requiring treatment & 2.6 \\
\hline TIA & 0.6 \\
\hline CVA & 0.3 \\
\hline Dementia & 1.4 \\
\hline Coma & 0.3 \\
\hline Respiratory failure & 2.0 \\
\hline Chest tube $>3 \mathrm{~d}$ & 3.2 \\
\hline Renal failure & 0.6 \\
\hline Reoperation for bleeding & 0.9 \\
\hline Wound dehiscence & 1.7 \\
\hline Mediastinitis & 0.6 \\
\hline Superficial wound infection & 3.5 \\
\hline
\end{tabular}

OR, Operating room; $M I$, myocardial infarction; $C H F$, congestive heart failure; TIA, transient ischemic attack; $C V A$, cerebrovascular accident.

protocol called for CABG for those with the most extensive disease. Although regional and institutional differences were present, overall, the ratio of $\mathrm{PCI}$ to $\mathrm{CABG}$ procedures of 2:1 was close to most centers' experience. The higher number of lesions and increased myocardium at risk for the CABG strata also reflected the design of the trial. ${ }^{4,5}$

Although BARI 2D was a negative trial when analyzing the entire cohort in terms of both any advantage of early revascularization and the control of glycemia by insulin sensitization, the analysis by predefined strata showed an advantage for CABG relative to medical therapy in reducing the combined outcome of death, MI, and stroke $(22.4 \%$ CABG vs $30.5 \%$ medical therapy, $P<.001)$ and MI, in particular. This was the first demonstration of a reduction in the rate of $\mathrm{MI}$ in stable patients in any 
BARI 2D Assigned CABG 30-day Event Rates $(\mathrm{N}=347)$

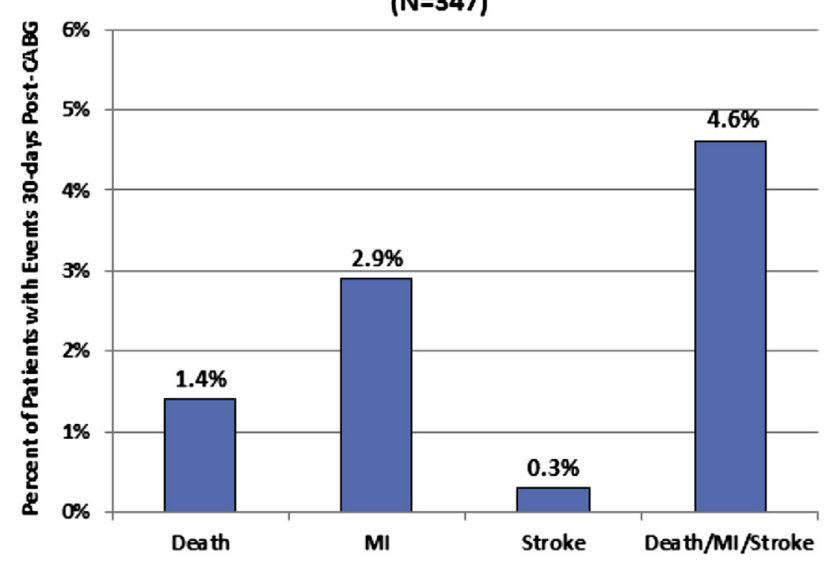

FIGURE 1. The percentage of patients who experienced death, myocardial infarction $(M I)$, or stroke in the 30 days after the assigned coronary artery bypass grafting procedure in Bypass Angioplasty Revascularization Investigation 2 Diabetes (BARI 2D) trial. The MIs were classified by the BARI 2D Electrocardiographic Core Laboratory, and the strokes were adjudicated by a centralized events classification committee. $C A B G$, Coronary artery bypass grafting.

randomized trial of revascularization compared with medical management. This reduction in the rates of MI was observed only in those with the most extensive CAD. ${ }^{6}$ To place this finding in context, it is important to characterize the $\mathrm{CABG}$ group and describe how the procedure was performed and how the observed outcomes relate to those from other large series.

The 30-day mortality of $1.4 \%$ compared very well to the data reported by the Society of Thoracic Surgeons database. From 2000 to 2009, the range of observed 30-day mortality for CABG-only procedures was $2.4 \%$ to $1.9 \%{ }^{7}$. This also compared favorably to historic data in which the presence of DM was thought to increase the 30-day mortality. The rates reported regarding surgery done in the late 1990s have ranged from $3.7 \%$ to $3.9 \%{ }^{8,9}$ In addition, the perioperative morbidity, including neurologic injury, renal failure, and reoperation for bleeding and postoperative infection, was low and comparable to that from the larger database. Regarding the conduct of $\mathrm{CABG}$, more than one third of the patients underwent off-pump CABG. For those done on pump, the bypass duration and crossclamp times were very acceptable, considering the number of grafts placed was 3, also the average number of grafts seen in most other large series. The types of myocardial protection used were also reasonable, just as was the incidence of IMA or other arterial graft use. In our series, IMA use was $94 \%$, the same reported by the Society of Thoracic Surgeons in 2009. As reported by other series, IMA use in the BARI 2D trial was associated with decreases in death, MI, and the combined death, MI, and stroke rate at 5 years. Overall, the 5-year mortality rate in the BARI 2D CABG cohort was $13.6 \%$. $^{2}$

\section{CONCLUSIONS}

We have previously demonstrated in the BARI 2D trial that CABG for patients with type $2 \mathrm{DM}$ with mild or no symptoms reduces the subsequent rates of MI in those with high myocardial jeopardy scores (ie, those with the most extensive disease). This result was achieved with the use of off-pump surgery in certain cases, adequate myocardial protection if $\mathrm{CABG}$ was performed on pump, routine use of an IMA or other arterial graft reflecting local experience at experienced centers, and systematic improvements in care that have reduced the influence of DM on CABG perioperative mortality.

\section{References}

1. The Bypass Angioplasty Revascularization Investigation (BARI) Investigators. Comparison of coronary artery bypass surgery with angioplasty in patients with multivessel disease. N Engl J Med. 1996;335:217-25.

2. Frye RL, August P, Brooks M, Hardison RM, Kelsey SF, MacGregor JM, et al; BARI IID Study Group. A randomized trial of therapies for type 2 diabetes and coronary artery disease. N Engl J Med. 2009;360:2503-15.

3. Alderman EL, Stadius M. The angiographic definitions of the Bypass Angioplasty Revascularization Investigation. Coron Artery Dis. 1992;2:1189-207.

4. Kim LS, King SB, Kent K. Factors related to the selection of surgical versus percutaneous revascularization in diabetic patients with multivessel coronary artery disease in the BARI 2D trial. J Am Coll Cardiol Intv. 2009;2:384-92.

5. Brooks M, Barsness G, Chaitman BR, Chung S, Faxon D, Felt F, et al; BARI IID Study Group. Baseline characteristics of patients with diabetes and coronary artery disease enrolled in the Bypass Angioplasty Revascularization Investigation 2 Diabetes (BARI 2D) trial. Am Heart J. 2008;156:528-36. e5.

6. Brooks M, Chaitman BR, Nesto RW, Hardison RM, Feit F, Gersh BJ, et al. Clinical and angiographic risk stratification and differential impact on treatment outcomes in the Bypass Angioplasty Revascularization Investigation 2 Diabetes (BARI 2D) trial. Circulation. 2012;126:2115-24.

7. El Bardissi AW, Aranki SF, Sheng S, O'Brien SM, Greenberg CC, Gammie JS. Trends in isolated coronary artery bypass grafting: an analysis of the Society of Thoracic Surgeons adult cardiac surgery database. J Thorac Cardiovasc Surg. 2012;143:273-81.

8. Thourani VH, Weintraub WS, Stein B, Gebhart SSP, Craver JM, Jones EL, et al. Influence of diabetes mellitus on early and late outcome after coronary artery bypass grafting. Ann Thorac Surg. 1999;67:1045-52.

9. Carson JL, Scholz PM, Chen AY, Peterson ED, Gold J, Schneider SH. Diabetes mellitus increases short-term mortality and morbidity in patients undergoing coronary artery bypass graft surgery. J Am Coll Cardiol. 2002;40: 418-23. 